\title{
Sustainable Double-Network Structural Materials for Electromagnetic Shielding
}

Qing-Fang Guan ${ }^{\dagger}$, Zi-Meng Han ${ }^{\dagger}$, Kun-Peng Yang ${ }^{\dagger}$, Huai-Bin Yang, Zhang-Chi Ling, Chong-Han Yin, Shu-Hong Yu*

Division of Nanomaterials and Chemistry, Hefei National Laboratory for Physical Sciences at the Microscale, Institute of Energy, Hefei Comprehensive National Science Center, CAS Center for Excellence in Nanoscience, Department of Chemistry, Institute of Biomimetic Materials \& Chemistry, Anhui Engineering Laboratory of Biomimetic Materials, University of Science and Technology of China, Hefei 230026, China. $\dagger$ These authors contributed equally to this work.

*Correspondence to: Shu-Hong Yu (email: shyu@ustc.edu.cn) 


\section{EXPERIMENTAL METHODS}

Fabrication of Cellulose Nanofiber (CNF) / Carbon Nanotubes (CNTs)

All reagents and raw materials were commercially available. Calculated amount of water-based CNTs (5.0 wt \%) and TEMPO-oxidized cellulose nanofiber (CNF, $4.5 \mathrm{wt} \%$ ) were fully mixed and stirred at room temperature for 15 minutes. They were then sprayed by $\mathrm{CaCl}_{2}$ solution $(0.1$ M) until gelled and rinsed in deionized water three times. The CNF/CNTs hydrogels were cut into sheets, stacked by hydrogel layer-by-layer (HLBL). Last, CNF/CNTs composite was hotpressed at $80^{\circ} \mathrm{C}$ with a pressure of $100 \mathrm{MPa}$ until it was completely dry.

\section{Characterization}

A field emission scanning electron microscope (Carl Zeiss Supra 40) was used to take the SEM images. Before observation, all specimens were sputtered with gold for 30 seconds under a constant current of $30 \mathrm{~mA}$. An X-ray diffractometer (PANalytical X'pert PRO MRD) equipped with $\mathrm{Cu} \mathrm{K} \alpha$ radiation $(\lambda=1.54056 \AA$ ) was used to get X-ray diffraction (XRD) data. A Bruker Dimension FastScan Atomic Force Microscope (AFM) was used to take AFM images.

\section{Electrical properties measurements}

A two-probe method with a multimeter at room temperature in air was taken to measure the electrical conductivity of the CNF/CNTs. The two opposite sides of all the specimens were covered with a layer of silver paste. The I-V curve was measured by an adjustable power supply at room temperature in air. A layer of sliver paste was evenly pasted on two opposite sides of the specimens as electrode pairs.

\section{Electromagnetic shielding effectiveness test}

TE 10 waveguide techniques were used to measure the EMI SE at the frequency of 8-13 GHz. The specimen size was about $60 \mathrm{~mm} \times 60 \mathrm{~mm} \times 3 \mathrm{~mm}$.

\section{Mechanical performance testing}

An Instron 5565A universal testing machine was used to perform three-point bending test according to ASTM D790-15e1 at a loading rate of $1.0 \mathrm{~mm} \mathrm{~min}^{-1}$ with a support span of 12.5 $\mathrm{mm}$. The specimen size was about $25 \mathrm{~mm} \times 2 \mathrm{~mm} \times 2 \mathrm{~mm}$. The applied loading direction was perpendicular to the platelets. An Instron CEAST 9340 drop hammer tester was used to perform the drop hammer impact test of CNF/CNTs. The hammer with $15 \mathrm{~kg}$ (total mass) weight was falling freely from $30 \mathrm{~cm}$ height, and the specimen size was about $50 \mathrm{~mm} \times 50 \mathrm{~mm} \times 2 \mathrm{~mm}$. 


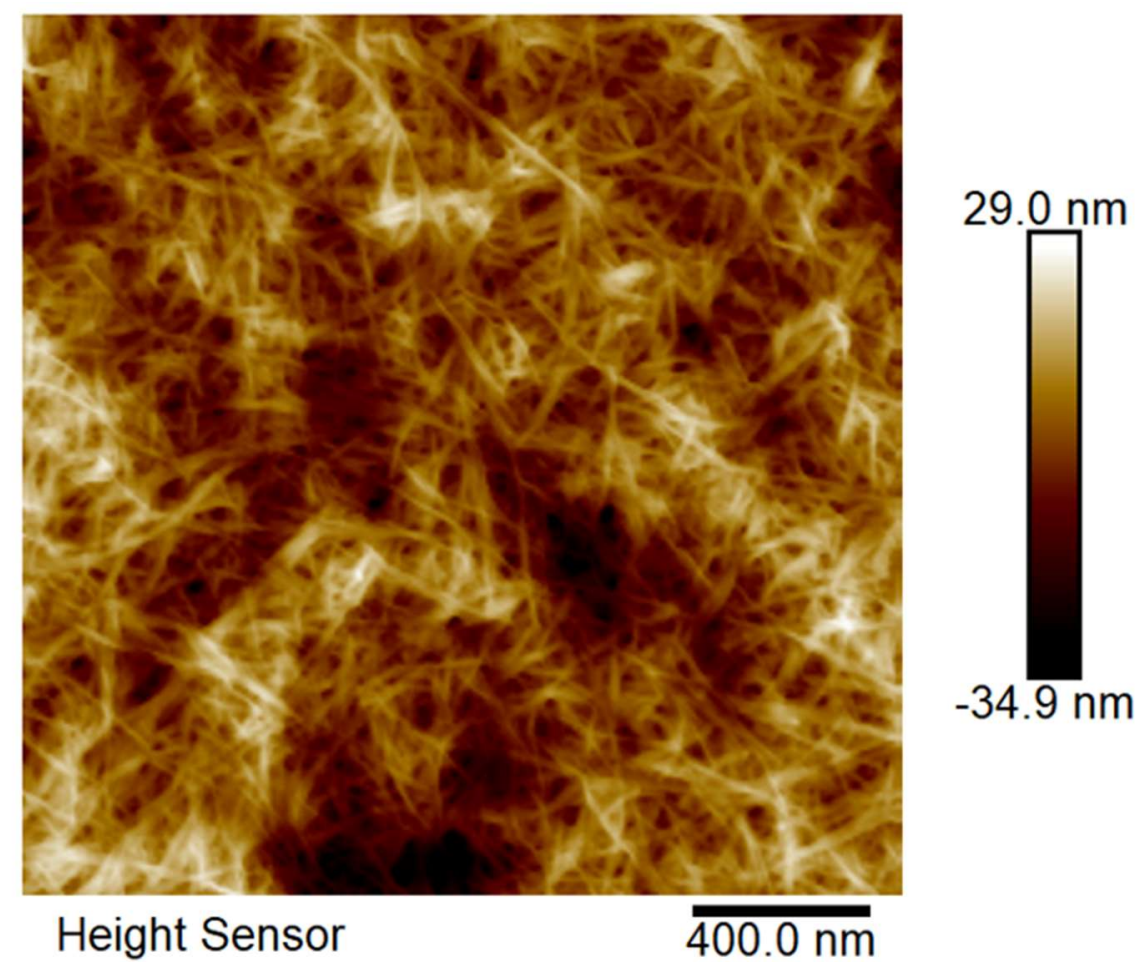

Supporting Figure S1. Atomic Force Microscope (AFM) image of Cellulose Nanofiber (CNF). 


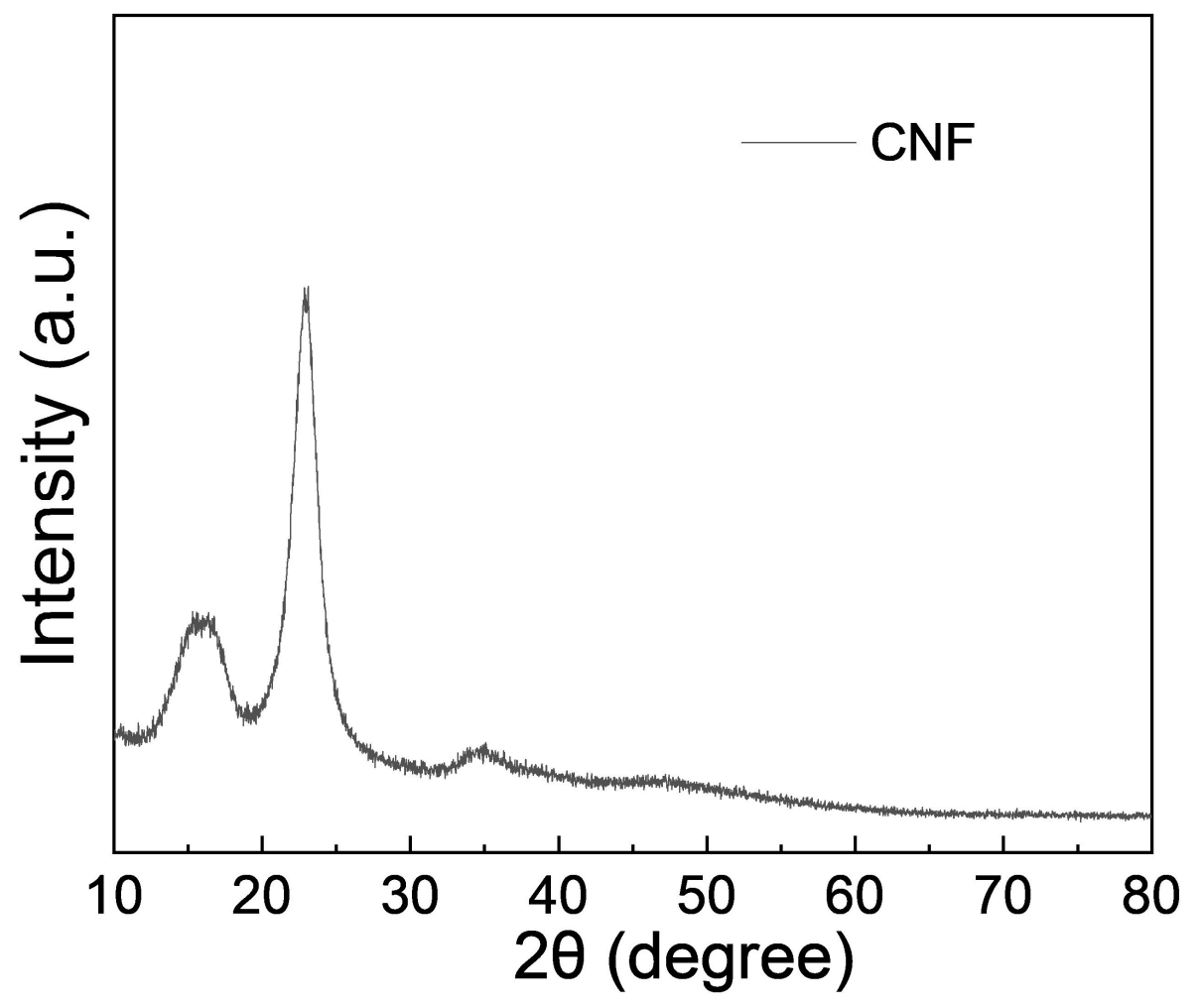

Supporting Figure S2. X-ray diffraction (XRD) pattern of CNF. 

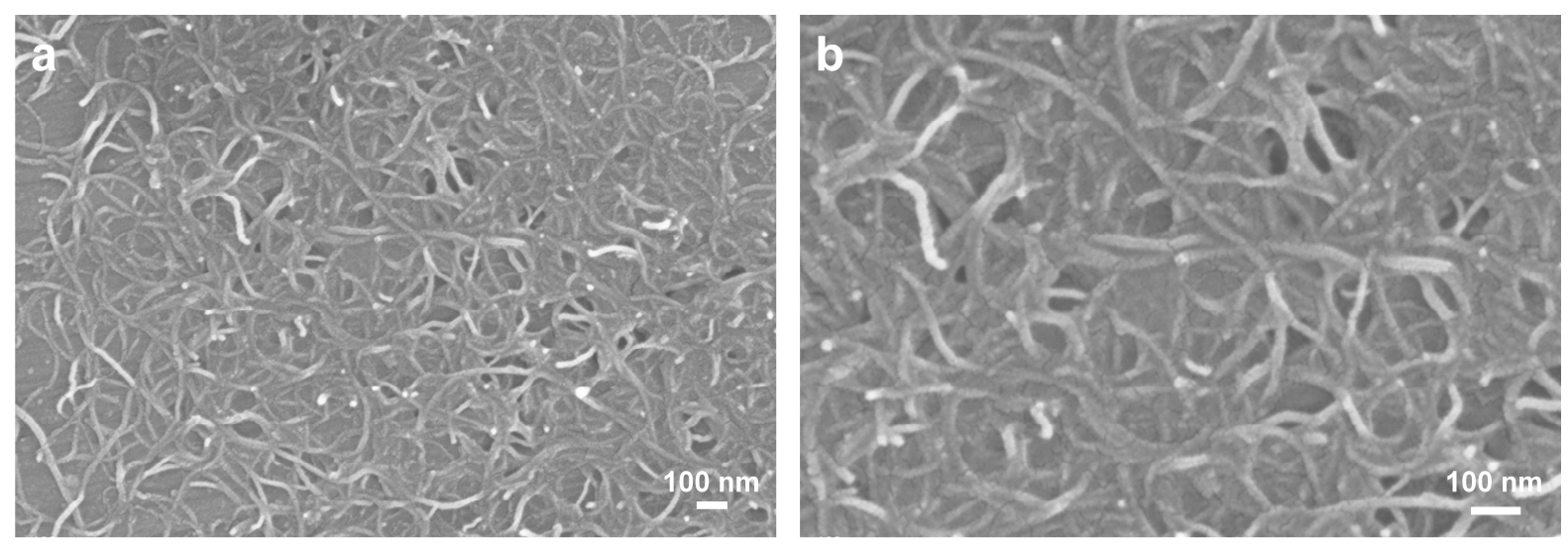

Supporting Figure S3. Scanning electron microscope (SEM) images of carbon nanotubes (CNTs). (a and b) SEM images of CNTs at different magnifications. 
a b
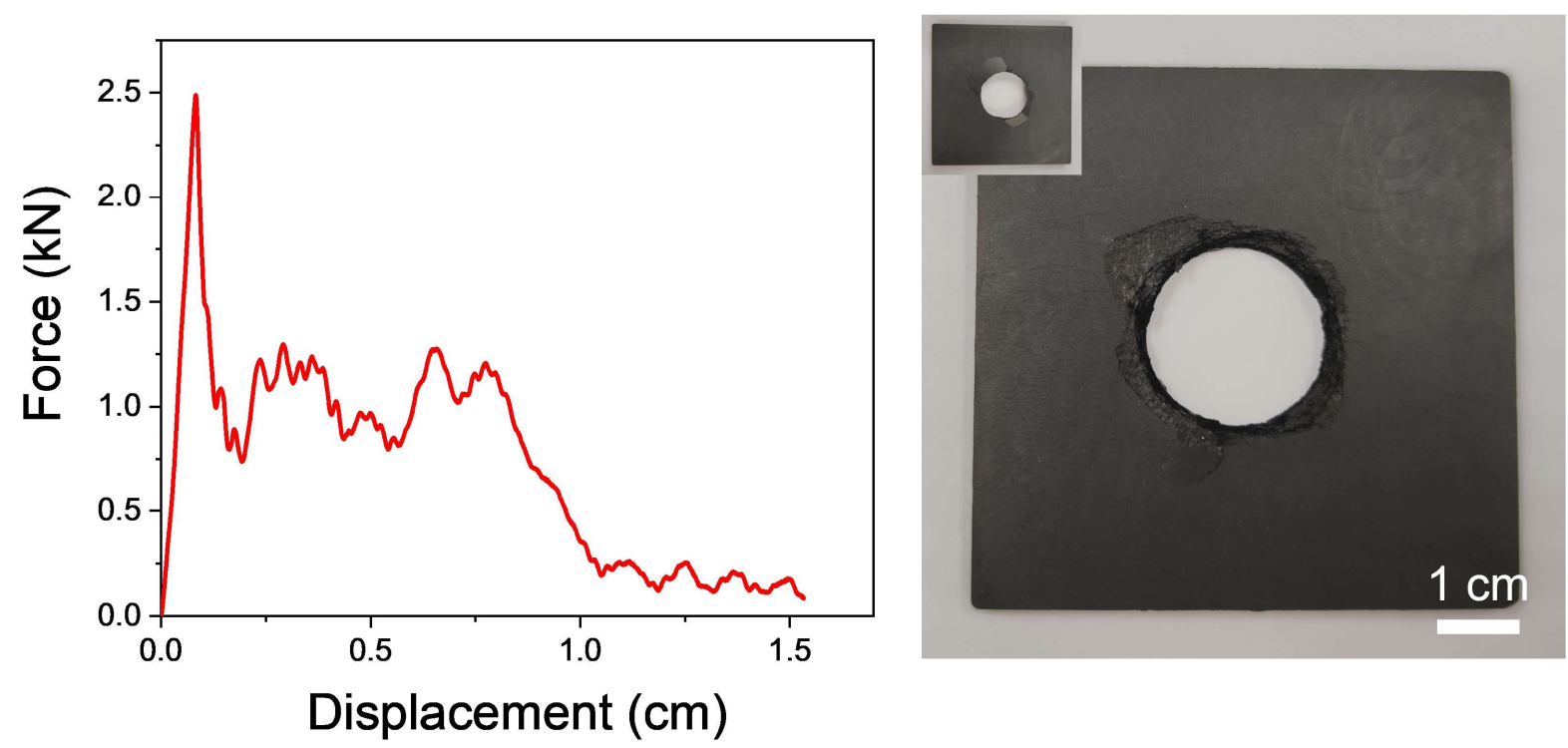

Displacement $(\mathrm{cm})$

Supporting Figure S4. Drop hammer impact test. (a) Force-displacement curve of CNF/CNTs

for the drop hammer impact test. (b) Photograph of CNF/CNTs after the drop hammer impact test. Scale bar, $1 \mathrm{~cm}$. 